
на популяционном уровне в Российской Федерации

\author{
Анна Васильевна Концевая ${ }^{1}$ Юлия Андреевна Баланова ${ }^{1 *}$, Асия Эмверовна Имаева1, \\ Михаил Борисович Худяков ${ }^{1}$, Олег Ильич Карпов², Оксана Михайловна Драпкина ${ }^{1}$ \\ ${ }^{1}$ Национальный медицинский исследовательский центр профилактической медицины \\ Россия, 101990, Москва, Петроверигский пер., 10 \\ ${ }^{2}$ Акционерное Общество «Санофи Россия». Россия, 125009, Москва, ул. Тверская, 22
}

Распространенность гиперхолестеринемии (ГХС) в Российской Федерации достаточно высока, и ассоциирована с клиническими последствиями и потенциальным экономическим ущербом, включающим не только и не столько затраты на ее коррекцию, сколько расходы на лечение заболеваний и осложнений, а также недополучение внутреннего валового продукта (ВВП).

Цель. Определить экономический ущерб от ГХС в российской популяции в 2016 г., включая прямые затраты системы здравоохранения и непрямые потери в экономике.

Материал и методы. Распространенность ГХС определена на основании отечественных популяционных исследований. В анализ включены российские статистические данные за 2016 г. по сердечно-сосудистым заболеваниям (ССЗ): ишемической болезни сердца (ИБС), инфаркту миокарда, цереброваскулярным сосудистым заболеваниям (многие цереброваскулярные заболевания не атеросклеротического генеза). Определен популяционный атрибутивный риск ГХС в развитии ССЗ во всей российской популяции. Использованы данные официальной статистики, показатели Программы государственных гарантий бесплатного оказания гражданам медицинской помощи.

Рассчитаны прямые и непрямые компоненты экономического ущерба от ГХС. Определено количество летальных исходов с расчетом «потерянных лет потенциальной жизни», недожитых до 72 летнего возраста. Потери, связанные с преждевременной смертностью в экономически активном возрасте, включали непроизведенный ВВП вследствие потерянных лет из-за смерти от ССЗ с учетом коэффициента занятости населения. Произведен расчет потерь ВВП вследствие инвалидности, исходя из числа лиц трудоспособного возраста со стойкой утратой трудоспособности в каждой из групп инвалидности и данных о ВВП на душу населения. Непрямые затраты из-за временной нетрудоспособности определены как выплаты заработной платы за дни нетрудоспособности, умноженные на количество дней по данным статистической Формы $16-\mathrm{BH}$.

Результаты. По данным расчетов среди обращений за медицинской помощью у больных ССЗ и ГХС на первом месте - амбулаторные, но по затратам системы здравоохранения - случаи госпитализации (затраты на оказание амбулаторной помощи 2,43 млрд руб, стационарной 18,21 млрд, руб). В структуре нозологий наибольшие затраты приходятся на ИБС, ассоциированную с ГХС. Прямые медицинские затраты составляют свыше 28,9 млрд руб, а с добавлением прямых немедицинских затрат - более 29,3 млрд руб в год. Потерянные годы потенциальной жизни составили свыше 1 млн лет в экономически активном возрасте. Основной экономический ущерб - свыше 1,29 трлн руб определяется непрямыми потерями в экономике, преимущественно потерями ВВП из-за преждевременной смертности в экономически активном возрасте (99\% ущерба). Совокупный экономический ущерб от ГХС в РФ определен в 1,295 трлн руб.

Заключение. Совокупный экономический ущерб от ГХС в РФ оставляет не менее 1,29 трлн руб в год, что эквивалентно 1,5\% ВВП (2016 г.). Прямые затраты, включающие затраты системы здравоохранения и выплаты пособий по инвалидности, составили лишь 2,3\% ущерба, в то время как 97,7\% - это потери в экономике вследствие преждевременной смертности и снижения производительности труда. Ожидается, что достижение целевых уровней контроля ГХС с помощью здорового образа жизни и лекарственной терапии должно снизить экономический ущерб.

Ключевые слова: гиперхолестеринемия, сердечно-сосудистые заболевания, экономический ущерб, затраты системы здравоохранения.

Для цитирования: Концевая А.В., Баланова Ю.А., Имаева А.Э., Худяков М.Б., Карпов О.И., Драпкина О.М. Экономический ущерб от гиперхолестеринемии на популяционном уровне в Российской Федерации. Рациональная Фармакотерапия в Кардиологии 2018; 14 (3):393-401. DOI: $10.20996 / 1819-6446-2018-14-3-393-401$

\title{
Economic Burden of Hypercholesterolemia in the Russian Federation
}

Anna V. Kontsevaya1', Yulia A. Balanova1*, Asia E. Imaeva1', Mihail B. Khudyakov', Oleg I Karpov², Oksana M. Drapkina

${ }^{1}$ National Medical Research Center for Preventive Medicine. Petroverigsky per., 10, Moscow, 101990 Russia

2 Public Company "Sanofi Russia". Tverskaya ul. 22, Moscow, 125009 Russia

Background. The prevalence of hypercholesterolemia (HCE) is quite high in the Russian Federation (RF), and it is associated with clinical consequences and with potential economic impact. Impact includes not only cost of its correction, but also the cost of treatment of diseases and complications, as well as the deficiency of the gross domestic product (GDP).

Aim. Evaluation of economic impact due to HCE in the Russian population, including direct expenditures of the Health Care System as well as nondirect impact in common economy.

Material and methods. Prevalence of HCE in the RF was identified based on local published studies. Local statistical data (2016) on cardio-vascular diseases (CVDs), including Ischemic Heart Disease (IHD), Myocardial Infarction and cerebral-vascular disease were included in the analysis. Population Attributive Risk (PAR) of HCE in CVDs has been extrapolated on all Russian population. Official statistics, parameters of Govern Guarantees Program of Free Medical Aid were used for modelling of direct and non-direct components of economic impact. Total amount of premature deaths with calculation of years of potential life lost until life expectation at 72 years was calculated. Economic impact due to premature CVDs mortality in economic activity age with consideration on ratio of employment have included GDP lost. Calculation of GDP lost also included monetary impact based on number of disability CVDs patients multiplied on GDP per capita in disability group. 
Results. Visits to policlinics of patients with CVDs and HCE had a first place among all calls for medical aid. In the same time, hospitalization required in higher expenditures (outpatients cost treatment expenditures were 2.43 billion RUR, in-patients treatment - 18.21 billion RUR). IHD with HCE was most expensive for direct expenditures in comparison with other CVD groups: more than 28.9 billion RUR per year, and with direct non-medical expenditures of 29.3 billion RUR in total. Years of potential life lost in economic active age were one million in total, 1.29 trillion RUR per year mostly due to indirect expenditures due to premature deaths in economy activity age (99\% of impact). Total Economic impact due to HCE in the Russian population for all HCE are estimated as 1.295 trillion RUR.

Conclusion. Total economic impact due to HCE in the RF is 1.5\% of GDP (2016), 1.295 trillion RUR. Direct expenditures included Health Care System expenses; disability covering had $2.3 \%$ only. Main part of impact is economic lost due to premature mortality and decrease of labor productivity. HCE patients control at target levels with help of healthy lifestyle and adequate pharmaceutical therapy can decrease economic impact.

Keywords: hypercholesterolemia, cardio-vascular diseases, economic burden, health care expenditures.

For citation: Kontsevaya A.V., Balanova Y.A., Imaeva A.E., Khudyakov M.B., Karpov O.I., Drapkina O.M. Economic Burden of Hypercholesterolemia in the Russian Federation. Rational Pharmacotherapy in Cardiology 2018;14(3):393-401. (In Russ). DOI: 10.20996/1819-6446-2018-14-3-393401

*Corresponding Author (Автор, ответственный за переписку): jbalanova@gnicpm.ru

Received / Поступила: 21.05.2018

Accepted / Принята в печать: 06.06.2018

Среди хронических неинфекционных заболеваний первое место во многих развитых странах занимают сердечно-сосудистые заболевания (ССЗ), причем, в Российской Федерации (РФ) они на первом месте и среди причин смертности, в том числе, и в трудоспособном возрасте, что ведет к значительному экономическому ущербу [1].

Доказано, что в основе многих ССЗ лежит атеросклероз, основой патогенеза которого являются нарушения липидного обмена - это повышение общего холестерина (ОХC), триглицеридов, холестерина (XC) липопротеидов низкой плотности в сыворотке крови, уровень ХС липопротеидов высокой плотности и комбинации этих нарушений. Вклад нарушений липидного профиля в смертность от ССЗ, ишемической болезни сердца и др. были показаны еще при анализе данных Фрамингамского исследованиия [2]. Крупное международное исследование INTERHEART продемонстрировало ведущую роль дислипидемии среди факторов риска инфаркта миокарда (ИМ) [3]

Высокая распространенность дислипидемии ассоциирована со значительными клиническими последствиями и с экономическим ущербом, включающим не только и не столько затраты на ее коррекцию, но и расходы на лечение заболеваний и осложнений, с ними связанных [4]. Ущерб может быть весьма значительным - это не только затраты на лечение, но и потери в экономике, связанные с инвалидностью, снижением производительности труда [5]. Именно ишемическая болезнь сердца (ИБС) как следствие гиперлипидемии является ведущей причиной инвалидности в Европе, обуславливая 10\% всех лет, прожитых в состоянии инвалидности [6].

В РФ актуальность нарушений липидного профиля особенного высока вследствие высокой их распро- страненности - более половины обследованных в популяционном исследовании лиц трудоспособного возраста имеют повышенный уровень ОХС [7,8]. Если сравнить распространенность гиперхолестеринемии (ГХС), полученную в популяционном исследовании «Эпидемиология сердечно-сосудистых заболеваний и факторов риска в некоторых регионах России» (ЭССЕ-РФ) с результатами программы мониторинга артериальной гипертонии, то очевидно, что частота этого показателя в стране возрастает [9] при низком охвате липидснижающей терапией [10].

В мире проводятся исследования по оценке экономического бремени дислипидемии и связанных с ней осложнений $[5,6]$. В нашей стране оценен совокупный ущерб от СС3 в целом без выделения его доли, обусловленной повышенным уровнем липидов [13]. Анализ экономического ущерба, ассоциированного с ГХС, является актуальным, поскольку может способствовать обоснованию, в том числе финансовому, мер, направленных на улучшение контроля данного фактора риска и снижение социально-экономического бремени ССЗ.

Цель работы: определить экономический ущерб от ГХС в российской популяции в 2016 г., включая прямые затраты системы здравоохранения и непрямые потери в экономике, обусловленные снижением производительности труда.

\section{Материал и методы}

Для определения распространенности ГХС были использованы данные популяционных исследований ЭССЕ-РФ [8] И МЕРИДИАН-РО [7].

В анализ были включены следующие ССЗ:

1. ИБС (код Международной классификации болезней [МКБ] 120-І25) 


\section{2. ИМ (код МКБ І21-22)}

3. Цереброваскулярные заболевания (ЦВЗ) (код МКБ 160-169)

\section{4. Ишемический инсульт (ИИ) (I63)}

Геморрагический инсульт (код МКБ - 160-162) был исключен на основании отсутствия риска, ассоциированного с ГХС.

Определили PAR (Population Attributive Risk, популяционный атрибутивный риск), отражающий частоту избыточной заболеваемости, обусловленной ГХС в популяции в целом, ГХС в развитии ССЗ, включенных в анализ. Объектом расчетов была вся популяция.

PAR - популяционный атрибутивный риск, определяется по следующей формуле:

$$
\text { PAR }=\frac{\mathrm{P}_{0}+\mathrm{P}_{1} \cdot R R-1}{\mathrm{P}_{0}+\mathrm{P}_{1} \cdot \mathrm{RR}} \text {. }
$$

Для $R R_{1}<1$, определяется PAR по редуцированной формуле:

$$
\mathrm{PAR}=\frac{\mathrm{P}_{1}+\mathrm{P}_{0} / R R-1}{\mathrm{P}_{1}+\mathrm{P}_{0} / \mathrm{RR}}
$$

где $\mathrm{P}_{1}$ - доля лиц в популяции лиц с факторами риска (ФР), Р - доля лиц без ФР, RR - (relative risk) относительный риск развития заболевания у лиц с ГХС на основании данных литературы (описаны далее в табл. 1).

PAR рассчитан для каждого заболевания (ИБС, ИМ, ЦВЗ, ИИ) и использован для определения доли заболеваемости/смертности, ассоциированных с ФР.

Основными источниками данных для настоящего исследования послужили:

- Данные федеральной службы государственной статистики (www.gks.ru), в том числе, половозрастная структура населения, повозрастная смертность насе- ления по кодам МКБ, экономические параметры (величина валового внутреннего продукта (ВВП), средней заработной платы и др.) за 2016 г.

- Данные Годовых форм федерального статистического наблюдения, в том числе, Ф.14 «Сведения о деятельности подразделений медицинской организации, оказывающих медицинскую помощь в стационарных условиях» (госпитализации, вызовы скорой медицинской помощи (СМП) по кодам МКБ-10), Ф.141 «Сведения о деятельности дневных стационаров медицинских организаций», Ф.12 «Сведения о числе заболеваний, зарегистрированных у пациентов, проживающих в районе обслуживания медицинской организации» и Ф.16-ВН «Сведения о причинах временной нетрудоспособности» (ВН) за 2016 г.

• Для определения стоимости амбулаторной и стационарной врачебной помощи использованы показатели Программы государственных гарантий оказания бесплатной медицинской помощи и соответствующих расчетных клинико-статистических групп на 2016 г. [14].

Компоненты экономического ущерба представлены на рис. 1. Под прямыми затратами понимали прямые медицинские затраты на амбулаторно-поликлиническую, стационарную (включая дневной стационар) и скорую медицинскую помощь (СМП), а под прямыми немедицинскими затратами понимали расходы на выплаты пособий по инвалидности.

Расчеты прямых медицинских затрат - на амбулаторно-поликлиническую, стационарную (включая дневной стационар) помощь и СМП - производились на основании количества госпитализаций, вызовов СМП и амбулаторных посещений по поводу ССЗ в 2016 г. (Ф. 12 и 14) с использованием стоимости од-

Table 1. Relative risk of developing adverse cardiovascular events included in calculations for 2016

Таблица 1. Относительный риск развития неблагоприятных сердечно-сосудистых событий, включенных в расчеты на 2016 г.

\begin{tabular}{|lccc|}
\hline Сердечно-сосудистые события & $\begin{array}{c}\text { ОР ГХС по сравнению } \\
\text { с нормальным уровнем ХС }\end{array}$ & Источник & PAR ГХС \\
\hline Смертность от ССЗ & 1,52 & {$[22,23]$} & 0,2329 \\
\hline Смертность от ИБС & 1,55 & {$[22,23]$} & 0,2431 \\
\hline Смертность от ИМ & 1,54 & {$[24]$} & 0,2398 \\
\hline Смертность от ЦВЗ & 1,77 & {$[25,26]$} & 0,3102 \\
\hline Смертность от инсульта & 1,77 & {$[25,26]$} & 0,3102 \\
\hline Заболеваемость ССЗ & 1,28 & {$[23]$} & 0,1393 \\
\hline Заболеваемость ИБС & 1,75 & {$[23]$} & 0,3046 \\
\hline Заболеваемость ИМ & 1,75 & {$[26]$} & 0,3046 \\
\hline Заболеваемость ЦВЗ & 1,35 & {$[23,27]$} & 0,1697 \\
\hline Заболеваемость Инсультом & 1,35 & 0,1697 \\
\hline РАR - Рориаатіо Аttributive Risk (популяционный атрибутивный риск), ОР - относительный риск, ГХС - гиперхолестеринемия, ХС - холестерин, & \\
ССЗ - сердечно-ссуудистые заболевания, ИБС - ишемическая болезнь сердца, ИМ - инфаркт миокарда, ЦВЗ - цереброваскулярные заболевания & \\
\hline
\end{tabular}




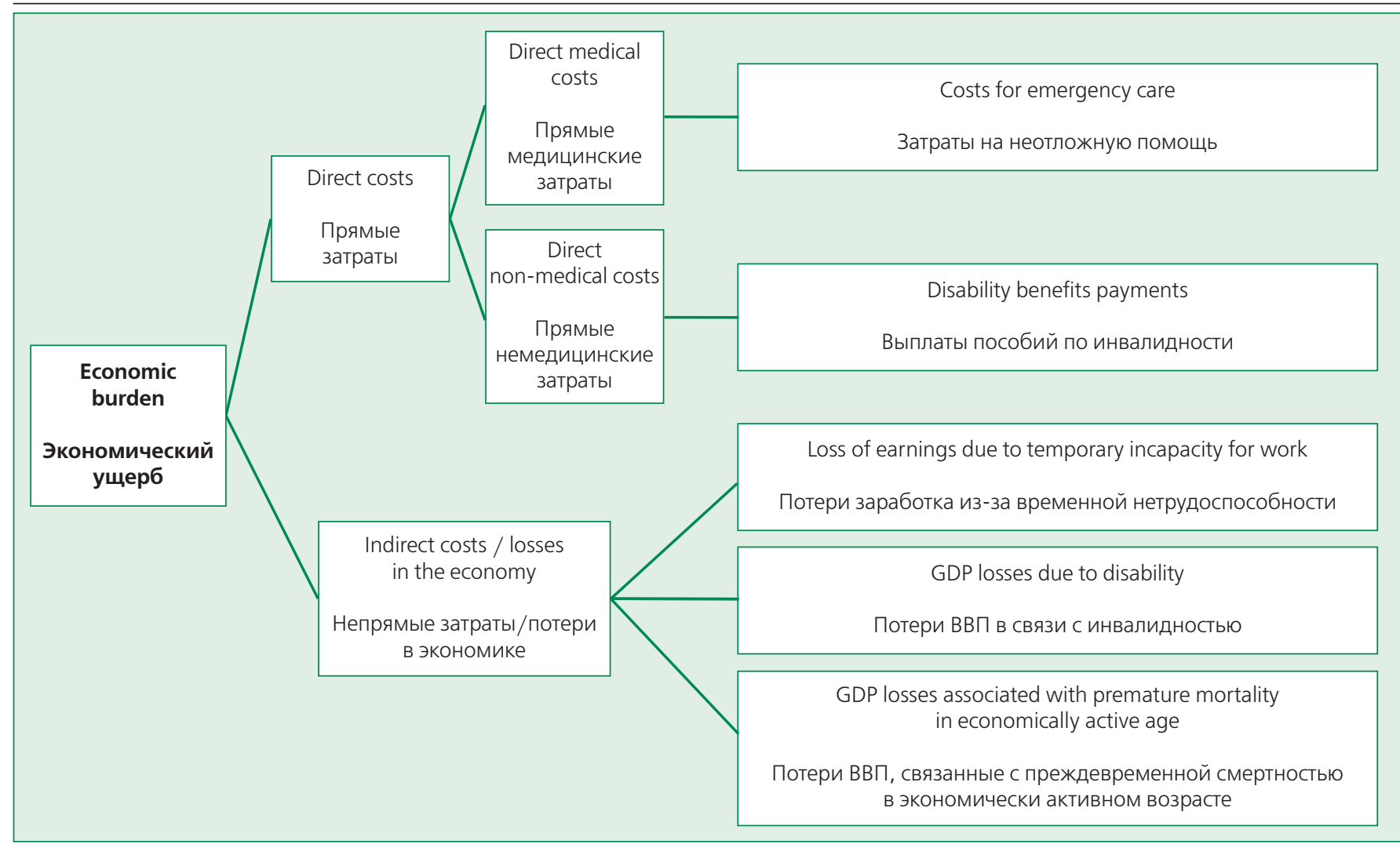

Figure 1. Components of the economic burden

Рисунок 1. Компоненты экономического ущерба

ного обращения/госпитализации по Программе государственных гарантий [14]. Тарифы, использованные для расчета стоимости госпитализаций при ИМ и инсульте, включенные в анализ, были детально описаны ранее [13]. Объем выплат пособий по инвалидности - прямые немедицинские затраты - рассчитывали по количеству инвалидов каждой группы для ССЗ [15] и по величине пособия по инвалидности [16].

Непрямые затраты (экономические потери) включали недополученный ВВП вследствие преждевременной смертности и инвалидности в экономически активном возрасте и потери заработка из-за ВН.

Смертность изучали по базе данных ВОЗ - WHO mortality database [17] и данным Российской базы данных по рождаемости и смертности [18]. Определяли абсолютное количество летальных исходов (повозрастная смертность от конкретной причины на 1000000 населения умноженная на численность населения соответствующего возраста и пола) и рассчитывали показатель «потерянные годы потенциальной жизни» (ПГПЖ) - число лет жизни, недожитых в экономически активном возрасте вследствие преждевременной смерти - путем суммирования абсолютного числа смертей в каждой возрастной группе с умножением на число лет, недожитых до 72 лет для каждой возрастной группы. Потери, связанные с преждевременной смертностью в экономически активном воз- расте, включали непроизведенный ВВП вследствие потерянных лет жизни в соответствующей возрастной группе вследствие смерти от ССЗ с учетом коэффициента занятости населения [19]. Потери будущих лет рассчитывали с использованием подхода «net present value/чистой приведенной стоимости» будущих потерь с 3\% дисконтированием [20].

Потери ВВП вследствие инвалидности определены следующим образом: вначале было рассчитано число лиц трудоспособного возраста со стойкой утратой трудоспособности в каждой из групп инвалидности. Затем расчетное число неработающих инвалидов трудоспособного возраста было умножено на ВВП на душу населения [21]. Непрямые затраты из-за временной нетрудоспособности вследствие ССЗ считали как выплаты заработной платы за дни нетрудоспособности, умноженные количество дней ВН по данным Формы 16-ВН за 2016 г.

Расчеты проводились при помощи приложения MS Excel 10.0 (Microsoft, CША).

\section{Результаты}

Для расчетов были отобраны относительные риски (OP, RR) из крупных мета-анализов последних лет (табл.1) [22-27], где за референсное значение принимался нормальный уровень XС. Показано, что наличие ГХС значимо - более чем наполовину - 
Table 2. Requests for medical care associated with hypercholesterolemia in the Russian population for 2016 Таблица 2. Обращения за медицинской помощью, ассоциированные с ГХС, в российской популяции за 2016 г.

\begin{tabular}{|lcccc}
\hline Параметр & $\begin{array}{c}\text { Количество } \\
\text { амбулаторных } \\
\text { обращений }\end{array}$ & $\begin{array}{c}\text { Количество } \\
\text { случаев } \\
\text { госпитализаций }\end{array}$ & $\begin{array}{c}\text { Количество } \\
\text { случаев } \\
\text { госпитализаций } \\
\text { в дневной стационар }\end{array}$ & $\begin{array}{c}\text { Количество } \\
\text { случаев } \\
\text { вызова СМП }\end{array}$ \\
\hline ССЗ & 4723420 & 681121 & 333727 & 217273 \\
\hline ИБС & 2326623 & 535579 & 164384 & 190063 \\
В том числе: инфаркт миокарда & 57031 & 63084 & 4029 & 45658 \\
\hline Цереброваскулярные болезни & 975622 & 202507 & 84010 & 74778 \\
из них: инсульт & 70254 & 71828 & 4964 & 52749 \\
\hline
\end{tabular}

ССЗ - сердечно-сосудистые заболевания, ИБС - ишемическая болезнь сердца, СМП - скорая медицинская помощь

Table 3. Disability, lost years of potential life and temporary disability associated with hypercholesterolemia in the Russian Federation in 2016

Таблица 3. Инвалидность, потерянные годы потенциальной жизни и временная нетрудоспособность, ассоциированные с ГХС в РФ в 2016 г.

\begin{tabular}{lccc}
\hline Параметр & Первичный выход на инвалидность & Число дней ВН & Число ПгпЖ \\
\hline ССЗ & 28825 & 3979619 & 1054787 \\
\hline ИБС & 14198 & 1496559 & 508593 \\
из них: инфаркт миокарда & 348 & 1183552 & 87573 \\
\hline ЦВЗ & 13023 & 1087155 & 304651 \\
из них: инсульт & 769 & 1029627 & 248270 \\
\hline
\end{tabular}

ССЗ - сердечно-сосудистые заболевания, ИБС - ишемическая болезнь сердца, ЦВЗ - цереброваскулярные заболевания, ВН - временная нетрудоспособность,

ПгПЖ - потерянные годы потенциальной жизни

увеличивает риск смерти от ССЗ, в том числе, ИБС и ИМ и ЦВЗ, в том числе - инсульта. Помимо увеличения риска смерти с ГХС также ассоциировано увеличение риска заболеваемости по вышеперечисленным нозологиям. На основании относительных рисков ГХС был рассчитан PAR ГXC. PAR ГХС в смертности составил от 23\% для ИБС до $31 \%$ для инсульта. PAR ГXС в заболеваемости также весьма существенен - от 14\% для ССЗ до 30\% для ИБС.

Детально было проанализировано количество обращений за медицинской помощью, ассоциированных с ГХС (табл. 2). На первом месте по количеству амбулаторные обращения, создающие серьезную нагрузку на амбулаторное звено системы здравоохранения. В структуре амбулаторных обращений, ассоциированных с ГХС, почти половину занимают связанные с ИБС. На втором месте - количество случаев госпитализации, преимущественно по ИБС.

В табл. 3 представлен показатель социального бремени - ПГПЖ, необходимый для определения непрямых потерь, ассоциированных с ГХС. В 2016 г. из-за преждевременной смерти, ассоциированной с ГХС, было потеряно свыше 1 млн лет потенциальной жизни в экономически активном возрасте в РФ. Для расчета непрямых потерь в экономике в анализ были включены: число дней временной нетрудоспособно- сти и число случаев выхода на инвалидность (табл. 3). С ГХС ассоциировано 28,8 тыс случаев первичного выхода на инвалидность по поводу ССЗ и почти 4 млн дней временной нетрудоспособности.

Суммарный экономический ущерб в российской популяции, связанный с ГХС у пациентов, имеющих СС3, детально представлен в табл. 4. Несмотря на то, что по количеству обращений амбулаторные посещения выходят на первое место, в структуре прямых медицинских затрат системы здравоохранения наибольшие затраты связаны с расходами на стационарную помощь (рис. 2). Вместе с тем по всем видам анализируемой медицинской помощи в структуре нозологий выделяются затраты на ИБС, ассоциированную с ГХС. Прямые медицинские затраты составляют свыше 28,9 млрд руб в 2016 г., а с добавлением прямых немедицинских прямые затраты - более 29,3 млрд руб (рис. 2).

Однако основной экономический ущерб - свыше 1,2 трлн руб - определяется непрямыми потерями в экономике, где на первое место выходят потери ВВП, связанные с преждевременной смертностью в экономически активном возрасте (рис. 3 ).

Таким образом, прямые затраты составляют 2,3\%, а непрямые потери в экономике - 97,7\%. Совокупный экономический ущерб, ассоциированный с ГХС у па- 
Table 4. Economic losses due to hypercholesterolemia in the population in patients with cardiovascular diseases according to nosologies (rubles) in 2016 in the Russian population

Таблица 4. Экономические потери в связи с ГХС в популяции у пациентов с ССЗ по нозологиям (руб) в 2016 г. в российской популяции

\begin{tabular}{|c|c|c|c|c|c|}
\hline \multirow[t]{2}{*}{ Параметр } & CC3 & ИБС & ИM & ЦВЗ3 & Инсульт \\
\hline & \multicolumn{5}{|c|}{ Прямые медицинские затраты системы здравоохранения } \\
\hline Амбулаторная помощь & 4938195659 & 2431786469 & 59609195 & 1019984122 & 73429230 \\
\hline Стационарная помощь & 23160241352 & 18206658891 & 4044297418 & 5313227910 & 5112906054 \\
\hline Дневной стационар & 441768389 & 5215466171 & 127844177 & 91247244 & 5391298 \\
\hline СМП & 379826581 & 332172685 & 79796482 & 130689663 & 92189275 \\
\hline \multicolumn{6}{|l|}{ Прямые медицинские затраты системы } \\
\hline \multirow[t]{2}{*}{ здравоохранения, всего } & 28920031981 & 26186084216 & 4311547272 & 6555148938 & 5283915856 \\
\hline & \multicolumn{5}{|c|}{ Прямые немедицинские затраты системы здравоохранения } \\
\hline Выплаты пособий по инвалидности & 429340674 & 96683438 & 2369954 & 88680299 & 5239631 \\
\hline \multirow[t]{2}{*}{ Все прямые затраты } & 29349372655 & 26282767654 & 4313917226 & 6643829238 & 5289155487 \\
\hline & \multicolumn{5}{|c|}{ Потери в экономике (непрямые затраты) } \\
\hline Временная нетрудоспособность & 6796541105 & 3709338413 & 2125164097 & 1856203324 & 1757980615 \\
\hline Потери ВВП от инвалидности & 5782989456 & 1302274247 & 31922013 & 1194476248 & 70575029 \\
\hline Потери ВВП от преждевременной смертности & 1253681097013 & 604495102617 & 104085873243 & 362097028336 & 295084684089 \\
\hline Непрямые затраты, всего & 1266260627574 & 609506715278 & 106242959354 & 365147707907 & 296913239733 \\
\hline Экономический ущерб полный & 1295610000228 & 635789482931 & 110556876580 & 371791537145 & 302202395220 \\
\hline В \% от ВВП в 2016 г. & $1,51 \%$ & $0,73 \%$ & $0,13 \%$ & $0,43 \%$ & $0,35 \%$ \\
\hline
\end{tabular}

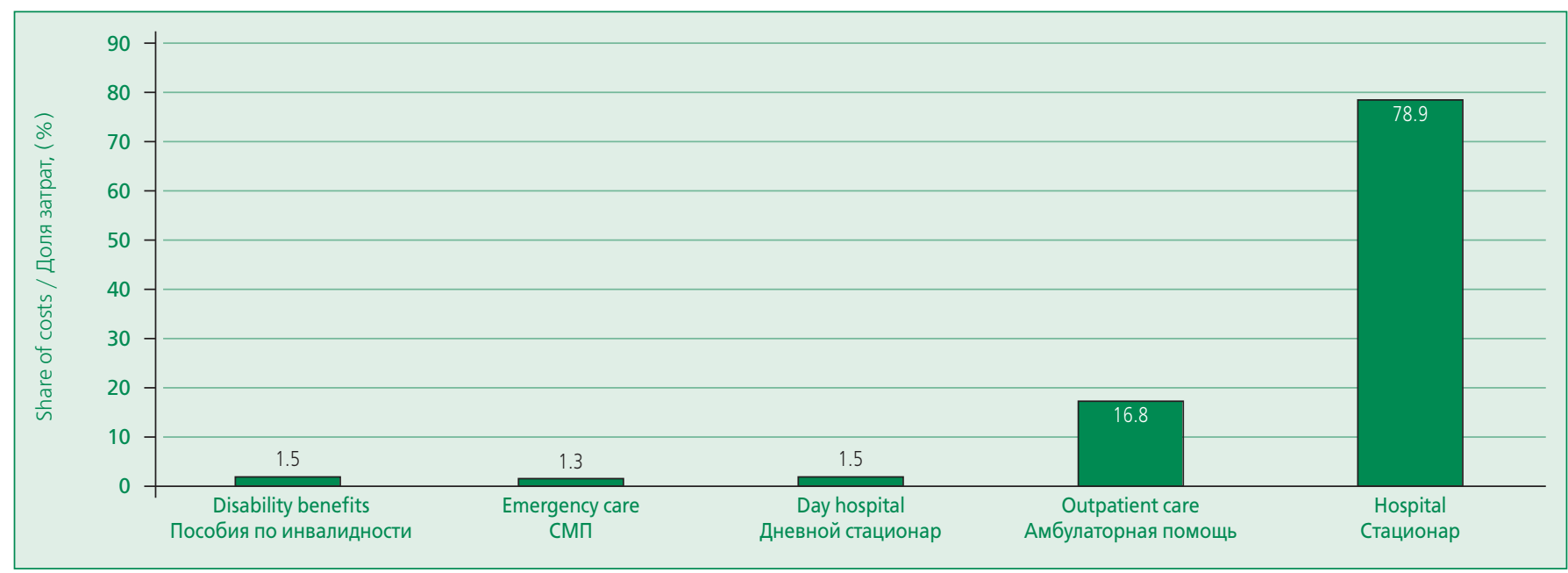

Figure 2. The structure of direct costs associated with hypercholesterolemia in patients with cardiovascular diseases in 2016 in the Russian population

Рисунок 2. Структура прямых затрат в связи с ГХС у пациентов с ССЗ в 2016 г. в российской популяции

циентов с ССЗ в РФ за 2016 г., составил около 1,3 трлн руб, что соответствует $1,51 \%$ ВВП.

\section{Обсуждение}

Дислипидемия является значимым фактором риска среди ССЗ во всем мире. Распространенность
ГХС в мире неоднородна. Barquera S. и соавт. в 2015 г. опубликовали крупный мета-анализ, показавший неоднородность значений ХС - если в Гане распространенность ГХС составляет 18,1\%, в Афганистане, Сенегале, Непале не превышает 25\%, то в Мексике, Чехии, Финляндии, США и Великобритании - свыше 


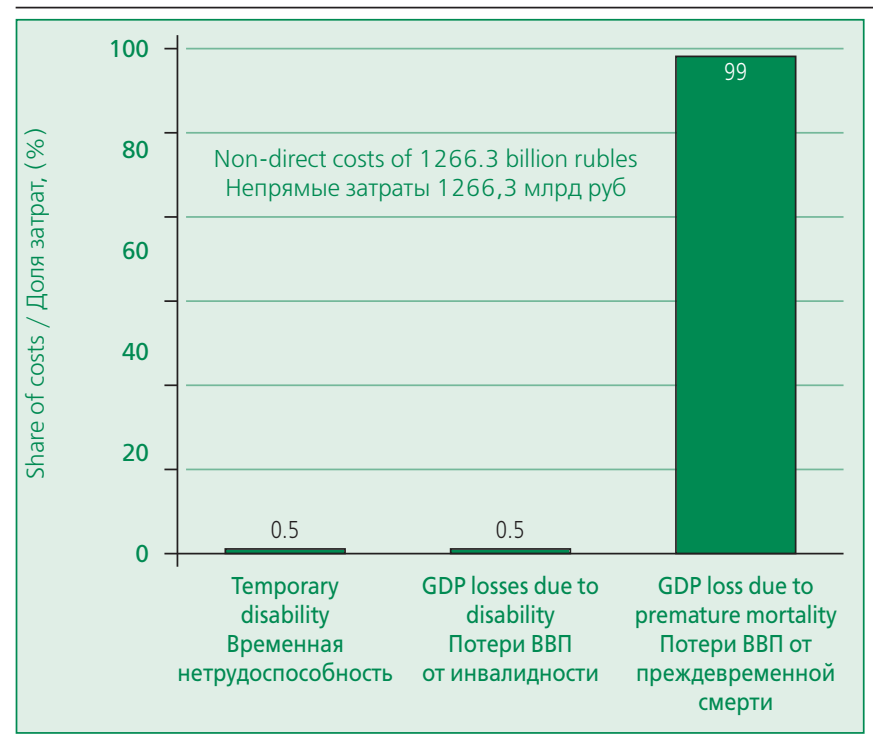

Figure 3. Structure of indirect costs associated with hypercholesterolemia in patients with cardiovascular diseases in 2016 in the Russian population

Рисунок 3. Структура непрямых затрат в связи с ГХС у пациентов с ССЗ в 2016 г. в российской популяции

50\% [28]. Среди жителей нашей страны доля лиц, имеющих отдельные нарушения липидного обмена, а также их комбинации достаточно высока [7-9, 29]. Распространенность повышенного уровня ОХС крови отмечается уже в детском и подростковом возрасте у 22\% девушек и 24\% юношей 15-19 лет, но также отмечено его снижение за период с 2003 по 2009 гг. [30]. В популяционном исследовании МЕРИДИАН-РО было показано, что среди жителей Рязанской области 35-64 лет частота ГХС (более 5 ммоль/л) составила 58,1\% [7]. Исследование ЭССЕ-РФ, анализировавшее данные представительной выборки россиян (свыше 20 тыс человек возраста 25-64 лет), выявило, что более половины обследованных имеют повышенный уровень ХС со значительными региональными колебаниями [8]. Вместе с тем среди посетителей лечебнопрофилактических учреждений, пришедших с любыми жалобами на прием к терапевту или кардиологу, этот показатель много выше - ГХС отмечается у $81,3 \%$ женщин и 78,9\% мужчин, как показали Ахмеджанов Н.М. и соавт. в исследовании АРГО [31].

За последние годы частота ГХС в нашей стране достоверно возросла, что подтверждает сравнение результатов программы «Мониторинг АГ», выполненной в первое десятилетие XXI века и ЭССЕ-РФ $[8,9]$. Противоположный результат показывают Rosinger A. и соавт. - в американской популяции отмечено достоверное снижение уровня ОХC [32]. Снижение уровня OХC отмечают и шведские исследователи проекта MONICA в период с 1994 по 2014 гг. среди лиц с высоким сердечно-сосудистым риском [33]. Австралий- ские исследователи также показали снижение уровня OXC в динамике с 1999 по 2013 гг. - с 5,13 до 4,53 ммоль/л за счет популяционных изменений образа жизни и, в большей степени, применения липидснижающей терапии [34].

Интерес исследователей и практических врачей к уровню липидов крови обоснован - McAloon и соавт., проанализировав причины смертей 17,6 млн умерших от ССЗ, установили, что ГХС повышает риск смерти от ИБС и инсульта и ассоциирована с 1/3 смертей от ИБС [35]. В исследовании Jousilahti Р. и соавт. обнаружено, что риск смерти от ИБС увеличивался почти в 3 раза у мужчин и в 4 раза у женщин с увеличением концентрации ХС выше 6,5 ммоль/л [36]. еще в 1999 г. Южноафриканские авторы показали, что 59\% случаев ИБС и 29\% ишемических инсультов обусловлены ГХС, а, следовательно является значимой причиной смертности и инвалидности [37]. Capewell S. и соавторы показали, что снижение распространенности ГХС на 5\% приводит к снижению смертности в популяции на 5\% [38]. Вместе с тем связь уровня ХС и смертности от ИБС в популяции лиц старшей возрастной группы не так однозначна. Так, в 2007 г. был проведен мета-анализ 61 исследования (около 892000 участников), посвященный оценке связи XС, артериального давления и сосудистой смертности [39]. Обнаружено, что связь уровня ОХС с развитием ИБС наиболее значима у лиц моложе 65 лет, тогда как в более старшем возрасте такая взаимосвязь слабая; вероятно, это связано с «эффектом выживаемости». Показано, что у лиц в возрасте моложе 60 лет наличие концентрации ОХС 5-6 ммоль/л, 68ммоль/л и > 8 ммоль/л в крови повышали риск возникновения ИБС в 2, 3 и 5 раз, соответственно, тогда как у лиц 60-70 лет относительный риск для концентрации 6-8 ммоль/л и > 8 ммоль/л составил 1,3 и 2,3, соответственно [39].

Доказано, что прием статинов, препаратов, снижающих снижающие концентрацию ОХС и XС липопротеидов низкой плотности в крови, уменьшает риск возникновения ССЗ и смертность от них, в том числе, инфарктов и инсультов [40-42]. Тем больше настораживает тот факт, что осведомленность об уровне ОХС крови в российской популяции крайне невысока только пятая часть мужчин и треть женщин среди 2564-летних знают свой уровень ХС, а 13,6\% мужчин и 18,2\% женщин знали о наличии у них повышенного уровня ОХС. Прием липидснижающих препаратов в России удручающе низок даже среди пациентов высокого и очень высокого риска [10]. Важно понимать, что частота нарушений липидного обмена с возрастом увеличивается, что подтверждают и российские данные [8]. В условиях демографического старения популяции в РФ общее число лиц с этими нарушениями 
будет возрастать, увеличивая затраты системы здравоохранения и государства в целом.

Затраты системы здравоохранения, ассоциированные с сердечно-сосудистыми событиями у лиц с ГХС, продемонстрированы в ряде исследований $[11,12,43]$. Так, португальские исследователи показали, что в ценах 2013 г. прямые затраты, ассоциированные с ГХС, составили $€ 320$ млн, а непрямые, связанные с выплатами по инвалидности - €198 млн [44]. В немецком исследовании так же, как в нашей работе, было показано, что наиболее значимым компонентом экономического ущерба от ГХС являются непрямые потери в экономике, обусловленные ранним выходом на инвалидность и другими факторами [45].

\section{Заключение}

Настоящее исследование является первым исследованием, оценившим экономический ущерб от ГХС в России. Показано, что совокупный экономический ущерб от ГХС в российской популяции в 2016 г. на фоне крайне низкого приема гиполипидемических препаратов составил 1,29 трлн руб, что эквивалентно 1,5\% ВВП страны в ценах 2016 г., причем, большая часть этого ущерба обусловлена ИБС. Прямые затраты, включающие затраты системы здравоохранения и выплаты пособий по инвалидности, составили лишь малую долю ущерба, в то время как 97,7\% - это потери в экономике вследствие преждевременной смертности и снижения производительности труда.

\section{References / Литература}

1. Oganov R.G., Kontsevaya A.V., Kalinina A.M. State Economic Burden of Cardiovascular Diseases in the Russian Federation. Cardiovascular Therapy and Prevention. 2011;10(4):4-9. (In Russ.) [Оганов Р.Г, Концевая А.В., Калинина А.М. Экономический ущерб от сердечно-сосудистых заболеваний в Российской Федерации. Кардиоваскулярная Терапия и Профилактика. 2011;10(4):4-9]

2. Mahmood S.S., Levy D., Vasan R.S. et al. The Framingham Heart Study and the epidemiology of cardiovascular disease: A historical perspective. Lancet. 2014;383:999-1008. doi 10.1016/501406736(13)61752-3

3. Yusuf, S., Hawken, S., Ôunpuu, S., et al. Effect of potentially modifiable risk factors associated with myocardial infarction in 52 countries (the INTERHEART study): case-control study. Lancet. 2004;364(9438):937-52. doi: 10.1016/S0140-6736(04)17018-9.

4. Dragomir A., Côté R., White M. et al. Relationship between Adherence Level to Statins, Clinical Issues and Health-Care Costs in Real-Life Clinical Setting. Value in Health. 2010;13(1):87-94. doi: 10.1111/j.1524-4733.2009.00583.x.

5. Virtanen M., Ervasti J., Mittendorfer-Rutz E., Lallukka T. et al. Work disability before and after a major cardiovascular event: A ten-year study using nationwide medical and insurance registers. Scientific Reports. 2017;7(1):1142. doi: 10.1038/s41598-017-01216-2.

6. National Institute for Health and Clinical Excellence (NICE). Statins for the prevention of cardiovascular events. [cited Jun 15, 2018]. Available from: http://www.sefap.it/servizi_lineeguida_200711/ NICE_TA094guidance.pdf.

7. Filippov E.V., Balanova Yu.A. Dyslipidemia and its relationship with chronic non-communicable diseases (according to the MERIDIAN-RO study). Medicinskij Sovet. 2015;12:104-10. (In Russ.) [Филиппов Е.В., Баланова Ю.А. Дислипидемии и их связь с хроническими неинфекционными заболеваниями (по данным исследования МЕРИДИАН-РО). Медицинский Совет. 2015;12:104-10].

8. Metelskaya V.A., Shalnova S.A., Deev A.D. Analysis of atherogenic dyslipidemias prevalence among population of Russian Federation (results of the ESSE-RF Study). Profilakticheskaya Meditsina. 2016;19(1):15-23. (In Russ.) [Метельская В.А., Шальнова С.А., Деев А.Д. и др. Анализ распространенности показателей, характеризующих атерогенность спектра липопротеинов, у жителей Российской Федерации (по данным исследования ЭССЕ-РФ). Профилактическая Медицина. 2016;19(1):15-23]. doi: 10.17116/profmed201619115-23.

\section{Ограничения исследования}

Анализ популяционной атрибутивной фракции ГХС проводится на основании относительных рисков, полученных в зарубежных исследованиях, так как российских популяционных проспективных исследований в масштабах страны в доступной литературе не найдено. В российской популяции относительные риски сердечно-сосудистых событий могут отличаться. Кроме того, ИБС в РФ и понимание этого заболевания, включая коды по МКБ в зарубежных исследованиях, могут отличаться. При появлении данных проспективных исследований на российской популяции достаточной продолжительности целесообразно произвести повторный анализ. ИБС - это гетерогенная группа заболеваний, не все клинические формы которой ассоциированы с ГХС. В силу связанных с относительными рисками ограничений, представленных в исследованиях, и имеющимися статистическими показателями, в анализ включена ИБС в целом.

Конфликт интересов. Обработка данных исследования проведена при поддержке компании АO «Санофи Россия» (Москва), что никоим образом не повлияло на результаты и собственное мнение авторов.

Disclosures. The processing of the survey data was performed with the support of the Public Company "Sanofi Russia" (Moscow), which in no way affected the results and the authors' own opinion.

9. Balanova Yu.A., Deev A.D., Ivanov V.M. The results of the first stage of the epidemiological situation monitoring of arterial hypertension in the Russian Federation (2003-2004), carried out in the framework of the federal target program "Prevention and treatment of hypertension in the Russian Federation". Moscow: Meditsina dlya vas; 2005. (In Russ.) [Баланова Ю.А., Деев А.Д., Иванов В.М., др. Результаты первого этапа мониторинга эпидемиологической ситуации по артериальной гипертонии в РФ (2003-2004), проведенного в рамках Федеральной целевой программы «Профилактика и лечение артериальной гипертонии в РФ». Москва: Медицина для вас; 2005].

10. Shalnova S.A., Deev A.D., Metelskaya V.A. et al. Awareness and treatment specifics of statin therapy in persons with various cardiovascular risk: the study ESSE-RF. Cardiovascular Therapy and Prevention. 2016;15(4):29-37. (In Russ.) [Шальнова С.А., Деев А.Д. Метельская В.А. и др. Информированность и особенности терапии статинами у лиц с различным сердечно-сосудистым риском: исследование ЭССЕ-РФ. Кардиоваскулярная Терапия и Профилактика. 2016;15(4):29-37]. doi: 10.15829/1728-8800-2016-4-29-37.

11. Hallberg S., Gandra S.R., Fox K.M. et al. Healthcare costs associated with cardiovascular events in patients with hyperlipidemia or prior cardiovascular events: estimates from Swedish populationbased register data. The European Journal of Health Economics. 2016;17(5):591-601. doi: 10.1007/s10198-015-0702-0.

12. Fox K.M., Wang L., Gandra S. R. et al. Clinical and economic burden associated with cardiovascular events among patients with hyperlipidemia: a retrospective cohort study. BMC cardiovascular disorders. 2016;16(1):13. doi: 10.1186/s12872-016-0190-x.

13. Kontsevaya A.V., Drapkina O.M., Balanova Y.A. et al. Economic Burden of Cardiovascular Diseases in the Russian Federation in 2016. Rational Pharmacotherapy in Cardiology. 2018;14(2):156-66. (In Russ.) [Концевая А.В., Драпкина О.М., Баланова Ю.А. и соавт. Экономический ущерб от сердечно-сосудистых заболеваний в Российской Федерации в 2016 году. Рациональная Фармакотерапия в Кардиологии. 2018;14(2):156-66]. doi: 10.20996/1819-6446-2018-14-2156-166.

14. Government Decision № 1382 of 19 December 2015 «On the Programme of State guarantees for providing citizens of the Russian Federation with free medical care for 2016» (In Russ.) [Постановление Правительства РФ от 19 декабря 2015 г. № 1382 «О Программе государственных гарантий бесплатного оказания гражданам медицинской помощи на 2016»]. 
15. The demographic yearbook of Russia. Statistical handbook. Moscow: Rosstat; 2017. (In Russ.) [Дeмографический ежегодник России (2017). Статистический сборник. М.: Росстат; 2017].

16. Government Decision N247 of 18.03 .2015 "On the approval of the indexation coefficient from 01/04/2015 of social pensions". [cited Jun 15, 2018]. Available from: http://base. garant.ru/70913974/. (In Russ.) [Постановление Правительства РФ от 18.03.2015 N 247 «06 утверждении коэффициента индексации с 01.04.2015 социальных пенсий». [цитировано 15.06.2018]. Доступно на: http://base.garant.ru/70913974/].

17. WHO Mortality Database [cited Jun 15, 2018]. Available from: http://www.who.int/healthinfo/ mortality_data/en/.

18. Russian database on fertility and mortality. [cited Jun 15, 2018]. Available from: http://demogrnes.ru/ru/demogr_indicat/data_description. (In Russ.) [Российская база данных по рождаемости и смертности (РосБРиС). [цитировано 15.06.2018]. Доступно на: http://demogr.nes.ru/ru/demogr_indicat/data_description].

19. Statistical Bulletin Labor and Employment in Russia. Moscow: Rosstat; 2017. (In Russ.) [Труд и занятость в России (2017). Статистический сборник. Москва: Росстат; 2017].

20. Hall P.S., Edlin R., Kharroubi S. et al. Expected net present value of sample information: From burden to investment. Medical Decision Making. 2012;32(3):E11-E21. doi: 10.1177/0272989X 12443010.

21. Government Decision №192/323H/45H/113 of 10 April 2012 "On the approval of the methodology for calculating economic losses from mortality, morbidity and disability of the population" №192/323H/45H/113 «On the approval of the Methodology for calculating economic losses from mortality, morbidity and disability of the population». [cited Mar 02, 2018]. Available from: http://www.garant.ru/products/ipo/prime/doc/70070542/. (In Russ.) [Приказ МЭР РФ, М3и СР РФ, Минфина РФ и Федеральной службы государственной статистики от 10 апреля 2012 г. № 192/323н/45н/113 «0б утверждении Методологии расчета экономических потерь от смертности, заболеваемости и инвалидизации населения» [цитировано 02.03.2018]. Доступно на: http://www.garant.ru/products/ipo/prime/doc/70070542/]

22. Gutierrez J., Ramirez G., Rundek T, et al. Statin Therapy in the Prevention of Recurrent Cardiovascular Events. Cochrane Database Syst Rev. 2013(1):909-19. doi: 10.1001/archinternmed.2012.2145.

23. Taylor F., Ward K., Moore T. H. et al. Statins for the primary prevention of cardiovascular disease ( Review ). Cochrane Rev. 2013(1):1. doi: 10.1002/14651858.CD004816.pub4.

24. Conly J., Clement F., Tonelli M., et al. Cost-effectiveness of the use of low- and high-potency statins in people at low cardiovascular risk. CMAJ. 2011;183(16):1189-202. doi: 10.1503/cmaj.101281.

25. Ford E.S., Capewell S. Trends in Total and Low-Density Lipoprotein Cholesterol among U.S. Adults: Contributions of Changes in Dietary Fat Intake and Use of Cholesterol-Lowering Medications. PLoS One. 2013;8(5):1-8. doi: 10.1371/journal.pone.0065228.

26. Guzman-Castillo M., Ahmed R., Hawkins N. et al. The contribution of primary prevention medication and dietary change in coronary mortality reduction in England between 2000 and 2007: a modelling study. BMJ Open. 2015;5(1):e006070. doi: 10.1136/bmjopen-2014-006070.

27. Wang W., Zhang B. Statins for the prevention of stroke: A meta-analysis of randomized controlled trials. PLoS One. 2014;9(3):e92388. doi: 10.1371/journal.pone.0092388.

28. Barquera S., Pedroza-Tobías A., Medina C. et al. Global Overview of the Epidemiology of Atherosclerotic Cardiovascular Disease. Archives of Medical Research. 2015;46(5):328-38. doi: 10.1016/j.arcmed.2015.06.006

29. Voevoda M.I., Kovalkova N.A., Ragino Yu.I. Prevalence of metabolic syndrome in 25-45-year-old Novosibirsk dwellers. Terapevticheskij Arkhiv. 2016;88(10):51-6. (In Russ.) [Воевода, М. И., Ковалькова, Н. А., Рагино, Ю. И., Травникова, Н. Ю., Денисова Д.В. Распространенность метаболического синдрома у жителей Новосибирска в возрасте от 25 до 45 лет. Терапевтический архив. 2016;88(10):51-6]. doi: 10.17116/terarkh2016881051-56.

\section{About the Authors:}

Anna V. Kontsevaya - MD, PhD, Deputy Director for Scientific and Analytical Work, National Medical Research Center for Preventive Medicine

Yulia A. Balanova - MD, PhD, Leading Researcher, Laboratory of Economic Analysis of Epidemiological Research and Preventive Technologies, Department of Epidemiology of Chronic Non-Communicable Diseases, National Medical Research Center for Preventive Medicine

Asia E. Imaeva - MD, PhD, Senior Researcher, Department of Epidemiology of Chronic Non-Communicable Diseases, National Medical Research Center for Preventive Medicine

Mihail B. Khudyakov - Leading Engineer, Laboratory of Economic Analysis of Epidemiological Research and Preventive Technologies, Department of Epidemiology of Chronic Non-Communicable Diseases, National Medical Research Center for Preventive Medicine

Oleg l. Karpov - MD, PhD, Professor, Head of Health Economics and Outcomes Research Group, Public Company "Sanofi Russia"

Oxana M. Drapkina - MD, PhD, Professor, Corresponding Member of the Russian Academy of Science, Director of National Medical Research Center for Preventive Medicine
30. Zvolinskaya E.Yu., Alexandrov A.A., Deev A.D. Epidemiological Situation With Main Risk Factors of Cardio-Vascular Diseases Among Young Adults in Russian Federation. Kardiologiia. 2015;55:126369. (In Russ.) [Зволинская Е.Ю., Александров А.А., Деев А.Д. и др. Эпидемиологическая ситуация по основным факторам риска развития сердечно-сосудистых заболеваний в Российской Федерации среди лиц молодого возраста. Кардиология. 2015;55:1263-69]. doi: 10.18565/cardio.2015.12.63-69.

31. Akhmedzhanov N.M., Nebieridze D.V., Safaryan A.S. et al. Analysis of hypercholesterolemia prevalence in the outpatient practice (according to the ARGO study): Part I. Ration Pharmacother Cardiol. 2015;11 (3):253-60. (In Russ.) [Ахмеджанов Н.М., Небиеридзе Д.В., Сафарян А.С. и др. Анализ распространенности гиперхолестеринемии в условиях амбулаторной практики (по данным исследования АРГО): Часть І. Рациональная Фармакотерапия в Кардиологии. 2015;11(3):253-60]. doi: 10.20996/1819-6446-2015-11-3-253-260.

32. Rosinger A. Carroll M. D., Lacher D., et al. Trends in Total Cholesterol, Triglycerides, and Low-Density Lipoprotein in US Adults, 1999-2014. JAMA Cardiol. 2016;2(3):339-41. doi: 10.1001/jamacardio.2016.4396.

33. Eriksson M., Forslund A.S., Jansson J.H., et al. Greater decreases in cholesterol levels among individuals with high cardiovascular risk than among the general population: The northern Sweden MONICA study 1994 to 2014. Eur Heart J. 2016;37(25):1985-92. doi: 10.1093/eurheartj/ehw052.

34. Chan T., Dabin B., Hyun K., et al. Falling cholesterol trend at acute coronary syndrome presentation is strongly related to statin use for secondary prevention. Elsevier Ireland Ltd; 2016;212:192-7. doi: 10.1016/j.jjcard.2016.03.038

35. McAloon C.J., Boylan L.M., Hamborg T., et al. The changing face of cardiovascular disease 2000 2012: An analysis of the world health organisation global health estimates data. Int J Cardiol. 2016;224:256-64. doi: 10.1016/j.ijcard.2016.09.026.

36. Jousilahti P, Vartiainen E, Pekkanen J et al. Serum Cholesterol Distribution and Coronary Heart Disease Risk : Observations and Predictions Among Middle-aged Population in Eastern Finland. Circulation. 1998;97(11):1087-94. 37. doi: 10.1161/01.CIR.97.11.1087.

37. Norman R., Bradshaw D., Steyn K., et al. Estimating the burden of disease attributable to high cholesterol in South Africa in 2000. S Afr Med J. 2007:97(8):708-15

38. Capewell S., Morrison C.E., McMurray J.J. Contribution of modern cardiovascular treatment and risk factor changes to the decline in coronary heart disease mortality in Scotland between 1975 and 1994. Heart. 1999;81:380-6. doi: 10.1136/hrt.81.4.380

39. Iversen A., Jensen J.S., Scharling H. et al. Hypercholesterolaemia and risk of coronary heart disease in the elderly: Impact of age. The Copenhagen City Heart Study. Eur J Intern Med. 2009;20(2):13944. doi: 10.1016/j.ejim.2008.06.003

40. Blackburn D.F., Dobson R.T., Blackburn J.L., et al. Cardiovascular morbidity associated with nonadherence to statin therapy. Pharmacotherapy. 2005;25(8):1035-43. doi: 10.1592/phco.2005. 25.8.1035.

41. Foody J.M., Joyce A.T., Rudolph A.E., et al. Cardiovascular outcomes among patients newly initiating atorvastatin or simvastatin therapy: A large database analysis of managed care plans in the United States. Clin Ther. 2008;30(1):195-205. doi: 10.1016/j.clinthera.2008.01.003.

42. Wei L., Wang J., Thompson P., et al. Adherence to statin treatment and readmission of patients after myocardial infarction: a six year follow up study. Heart. 2002;88(3):229-33. doi: 10.1136/heart. 88.3.229

43. Punekar R.S., Fox K.M., Richhariya A., et al. Burden of First and Recurrent Cardiovascular Events among Patients with Hyperlipidemia. Clin Cardiol. 2015;38(8):483-91. doi: 10.1002/clc. 22428.

44. Gouveia M., Borges M., Augusto M. et al. Cost and Burden Of Hypercholesterolemia In Portugal. Value Heal. 2014;17(7):A339. doi: 10.1016/j.jval.2014.08.668.

45. Müller-Nordhorn J., Englert H., Wegscheider K., et al. Productivity loss as a major component of disease-related costs in patients with hypercholesterolemia in Germany. Clin Res Cardiol. 2008;97(3):152-9. doi: 10.1007/s00392-007-0602-0

\section{Сведения об авторах}

Концевая Анна Васильевна - д.м.Н., первый зам. директора по научной и аналитической работе, НМИЦ ПМ

Баланова Юлия Андреевна - К.М.Н., В.Н.С., лаборатория экономического анализа эпидемиологических исследований и профилактических технологий, отдел эпидемиологии хронических неинфекционных заболеваний, НМИЦ ПМ

Имаева Асия Эмверовна - К.М.Н., С.Н.С., отдел эпидемиологии хронических неинфекционных заболеваний, НМИЦ ПМ

Худяков Михаил Борисович - ведущий инженер, лаборатория экономического анализа эпидемиологических исследований и профилактических технологий, отдел эпидемиологии хронических неинфекционных заболеваний, НМИЦ ПМ Карпов Олег Ильич - Д.м.н., профессор, руководитель группы по экономике здравоохранения, АО «Санофи Россия» Драпкина Оксана Михайловна - д.м.Н., профессор, член-корреспондент РАН, директор НМИЦ ПМ 